\title{
CARTESIAN AND LAGRANGIAN MOMENTUM
}

\author{
Alexander Afriat \\ ama51@pitt.edu
}

\begin{abstract}
Historical, physical and geometrical relations between two different momenta, characterized here as Cartesian and Lagrangian, are explored. Cartesian momentum is determined by the mass tensor, and gives rise to a kinematical geometry. Lagrangian momentum, which is more general, is given by the fiber derivative, and produces a dynamical geometry. This differs from the kinematical in the presence of a velocity-dependent potential. The relation between trajectories and level surfaces in Hamilton-Jacobi theory can also be Cartesian and kinematical or, more generally, Lagrangian and dynamical.
\end{abstract}

\section{Introduction}

What we now call “momentum” has theological roots, in Descartes' Principia philosophice (Pars secunda):

36. Deum esse primariam motus causam: et eandem semper motus quantitatem in universo conservare.

[...] Et generalem quod attinet, manifestum mihi videtur illam non aliam esse, quam Deum ipsum, qui materiam simul cum motu et quiete in principio creavit, jamque, per solum suum concursum ordinarium, tantumdem motus et quietis in ea tota quantum tunc posuit conservat. Nam quamvis ille motus nihil aliud sit in materia mota quam ejus modus; certam tamen et determinatam habet quantitatem, quam facile intelligimus eandem semper in tota rerum universitate esse posse, quamvis in singulis ejus partibus mutetur. Ita scilicet ut putemus, cum una pars materiæ duplo celerius movetur quam altera, et hæc altera duplo major est quam prior, tantundem motus esse in minore quam in majore; ac quanto motus unius partis lentior fit, tanto motum alicujus alterius ipsiæ qualis fieri celeriorem.

Conservation $^{1}$ follows from the perfection of the Creator; for how could the world have more or less motion than He first put into it. The amount of motion depends on size and celerity: a piece of matter has as much motion as another that moves twice as fast but is half as big. Tradition has, rightly or wrongly, turned these two features into mass and velocity, and adopted the term momentum. As we see no reason to depart from it (too much at any rate), Cartesian momentum will be taken to be something like the product of mass and velocity - which is what Newton has in his Definitio II:

\footnotetext{
${ }^{1}$ Here I summarize the most relevant ideas; but if a translation is preferred, the 'authorized' onewhich Descartes himself went through and corrected—is by the abbé Picot (1647): “36. Que Dieu est la premiere cause du mouuement, \& qu'il en conserue tous-jours vne égale quantité en l'vniuers. [...] Pour ce qui est de la premiere, il me semble qu'il est éuident qu'il n’y en a point d'autre que Dieu, qui de sa Toute-puissance a crée la matiere auec le mouuement \& le repos, \& qui conserue maintenant en l'vniuers, par son concours ordinaire, autant de mouuement \& de repos qu'il y en a mis en le creant. Car, bien que le mouuement ne soit qu'vne façon en la matiere qui est meuë, elle en a pourtant vne certaine quantité... qui n'augmente \& ne diminuë jamais..., encore qu'il y en ait tantost plus \& tantost moins en quelques vnes de ses parties. C'est pourquoi, lors qu'vne partie de la matiere se meut deux fois plus vite qu'vne autre, \& que cette autre est deux fois plus grande que la premiere, nous deuons penser qu'il y a tout autant de mouuement dans la plus petite que dans la plus grande; \& que toutesfois \& quantes que le mouuement d'vne partie diminuë, celuy de quelque autre partie ....augmente en proportion."
} 
Quantitas motus est mensura ejusdem orta ex velocitate et quantitate materice conjunctim.

Motus totius est summa motuum in partibus singulis; ideoque in corpore duplo majore, æquali cum velocitate, duplus est, \& dupla cum velocitate quadruplus. ${ }^{2}$

where, from Definitio I, "Quantitas materice est mensura ejusdem orta ex illius densitate et magnitudine conjunctim." 3 This momentum could be called Newtonian, for it is with Newton that it came out from the mists of Theology; but its Cartesian origins, however murky, should be remembered ...

Scattered through Lagrange's Mécanique analytique are (apparently nameless) ${ }^{4}$ partial derivatives like $\delta \Phi / \delta d \xi$, where $\Phi$ is a function ${ }^{5}$ of position and velocity, and the scalar $d \xi$ a velocity (component—along with, say, $d \psi, \ldots, d \omega$ ). Hamilton will write $\varpi_{i}=\delta T / \delta \eta_{i}^{\prime}$ or $m_{i} d x_{i} / d t=\delta V / \delta x_{i}$ and call it momentum, where $T$ is kinetic energy, $\eta_{i}^{\prime}$ a velocity and $V$ the characteristic function. But as the expression first appeared in Lagrange, the term Lagrangian momentum seems best.

\section{The two momenta}

So momentum, either way, is closely related to velocity. We can try to characterize the relationship in more modern terms by writing $p=\mu^{b} \dot{q}$. But what is $\mu^{b}$ ? Velocity $\dot{q}$ is a vector, in the linear space $T_{q} Q$ tangent at point $q$ to the configuration space ${ }^{6}$ $Q$, momentum $p$ a covector ${ }^{7}$ in the dual (cotangent) space $T_{q}^{*} Q$; so $\mu^{b}$, whatever it is, turns velocities in $T_{q} Q$ into momenta in $T_{q}^{*} Q ; \mu^{b}: T_{q} Q \rightarrow T_{q}^{*} Q$. The inverse $\mu^{\#}=\left(\mu^{b}\right)^{-1}$ (whose existence we assume) goes the other way; $\mu^{\#}: T_{q}^{*} Q \rightarrow T_{q} Q$.

\footnotetext{
${ }^{2}$ Which roughly says that "The quantity of motion is the measure of what is derived from the velocity and quantity of matter together. The motion of the whole is the sum of the motion in the single parts; and is therefore double in a body twice as big, with the same velocity, quadruple with twice the velocity."

${ }^{3}$ Roughly: "The quantity of matter is the measure of what is derived from its density and size together."

${ }^{4}$ A concept corresponding to our "momentum" seems to exist in Lagrange only to the extent that the expression quoted appears; I could find no name (not impulsion, not even quantité de mouvement) given to it.

${ }^{5}$ Typically $\Phi$ (which Lagrange naturally did not call the "Lagrangian") would have been the difference $T-V$ (in his notation) between what we now call the kinetic and potential energies. Since the potentials Lagrange considered depended only on position, so that $\delta V / \delta d \xi$ vanished, he often wrote $\delta T / \delta d \xi$. But the difference $T-V$ also figured as the integrand in Lagrange's variational principle, and there the potential is not irrelevant.

${ }^{6}$ Which is assumed to be a differential manifold; see Definition 1.4.1 in Abraham and Marsden (1978).

${ }^{7} \mathrm{~A}$ covector- the differential of a function, for instance - transforms vectors linearly into scalars. The transformation law for the components of a covector is the inverse of the law applying to those of a vector: as Hamilton's $\eta_{i}^{\prime}$ are the components of a vector, the $\varpi_{i}$ transform like a covector; and furthermore the $\delta V / \delta x_{i}$ are the components of the differential $d V$, which is a covector. Using more modern ideas, coordinates $p_{k}=\left\langle p, \partial / \partial q^{k}\right\rangle$ and $\dot{q}^{k}=\left\langle d q^{k}, \dot{q}\right\rangle$ (at some $q$ in $Q$ ) arise by specifying a suitably 'independent' set of (smooth) functions $q^{k}: Q^{\prime} \rightarrow \mathbb{R}$ defined on an appropriate subset $Q^{\prime} \subset Q$ containing $q$. The differentials $d q^{k}: T_{q} \mathbb{E} \rightarrow \mathbb{R}$ span the cotangent space $T_{q}^{*} \mathbb{E}$, and are dual to the vectors $\partial / \partial q^{k}=\left(d q^{k}\right)^{\sharp}$ spanning the tangent space $T_{q} \mathbb{E}(k=1, \ldots, M)$.
} 
But to begin with simpler cases. For a single free particle of unit mass moving in a Euclidean space $\mathbb{E}$, $\mu^{b}$ can be the (partially evaluated) ${ }^{8}$ twice-covariant metric tensor $e^{b}: T_{q} \mathbb{E} \rightarrow T_{q}^{*} \mathbb{E} \quad(\forall q \in \mathbb{E})$ that characterizes the geometry of $\mathbb{E}$, by turning vectors into covectors, velocities into momenta, and providing notions of angle and length. The squared length $\left\langle\dot{q}^{b}, \dot{q}\right\rangle=\left\langle e^{b} \dot{q}, \dot{q}\right\rangle$ is twice the kinetic energy $K$, where the velocity $\dot{q}$ belongs to $T_{q} \mathbb{E}$, the momentum $\dot{q}^{b}$ to $T_{q}^{*} \mathbb{E}$, and $\langle r, \dot{s}\rangle$ is the value of the covector $r$ at the vector $\dot{s}$. Even if a little more than mere multiplication by (unit) mass is involved, it seems reasonable to view $e^{b} \dot{q}$ as the Cartesian momentum. The Lagrangian momentum will be (the fiber derivative) $d L_{q}(\dot{q})$, where $L_{q}$ is the restriction $L_{q}=\left.L\right|_{T \mathbb{E}}$ of the Lagrangian ${ }^{9} L=K$ to the tangent space $T_{q} \mathbb{E}$, and the differential $d L_{q}(\dot{q}): T_{q} \mathbb{E} \rightarrow \mathbb{R}$ approximates $L_{q}$ linearly around $\dot{q} \in T_{q} \mathbb{E}$.

To go the other way $T_{q}^{*} \mathbb{E} \rightarrow T_{q} \mathbb{E}$ we need the energy

$$
E(q, \dot{q})=\left\langle\dot{q}^{b}, \dot{q}\right\rangle-L(q, \dot{q}): T \mathbb{E} \rightarrow \mathbb{R},
$$

defined on the tangent bundle ${ }^{10}$

$$
T \mathbb{E}=\bigcup_{q \in \mathbb{E}} T_{q} \mathbb{E},
$$

which is a differential manifold with twice as many dimensions as the underlying manifold (here the linear space $\mathbb{E}$ ). For the energy provides the Hamiltonian $H(q, p)=\left\langle p, p^{\sharp}\right\rangle-L(q, p): T^{*} \mathbb{E} \rightarrow \mathbb{R}$, which to every $\left(q, \dot{q}^{b}\right) \in T \mathbb{E}$ assigns the same value $H\left(q, \dot{q}^{b}\right)=E(q, \dot{q})$ that $E$ gives to $(q, \dot{q}) \in T \mathbb{E}$, where $p=\dot{q}^{b}, p^{\sharp}=\dot{q}$, and the cotangent bundle $T^{*} \mathbb{E}$ is the union

$$
\bigcup_{q \in \mathbb{E}} T_{q}^{*} \mathbb{E}
$$

(here the Cartesian product $T^{*} \mathbb{E}=\mathbb{E} \times \mathbb{E}^{*}$, where $\mathbb{E}^{*}$ is a space of covectors acting on its dual, $\mathbb{E}$ ). The differential $d H_{q}$ of the restriction $H_{q}=\left.H\right|_{T_{a}^{*} \mathbb{E}}$ takes us back to the tangent space: $d H_{q}(\cdot): T_{q}^{*} \mathbb{E} \rightarrow T_{q} \mathbb{E}$. The value $d H_{q}(p): T_{q}^{*} \mathbb{E} \rightarrow \mathbb{R}$ acts as a covector.

Here $\mu^{b}=m^{b}=d L_{q}$, so both momenta are the same, and we can write $p=\dot{q}^{b}=e^{b} \dot{q}=d L_{q}(\dot{q})$.

For a free particle of mass $m$ we have

\footnotetext{
8 The tensor $e^{\mathrm{b}}(\cdot): T_{q} \mathbb{E} \rightarrow T_{q}^{*} \mathbb{E}$ arises by specifying only one of the two arguments of $e(\cdot, \cdot): T_{q} \mathbb{E} \times T_{q} \mathbb{E} \rightarrow \mathbb{R}$. For instance $e(\dot{q}, \cdot): T_{q} \mathbb{E} \rightarrow \mathbb{R}$ turns a vector into a scalar.

${ }^{9}$ The Lagrangian is a function of position and velocity, in mechanics typically the difference between the kinetic and potential energies; without a potential it is just $K$.

${ }^{10}$ Here the tangent bundle is the Cartesian product $\mathbb{E} \times \mathbb{E}$, whose elements are ordered pairs of the form $(q, x)$, where $q$ belongs to the underlying manifold $\mathbb{E}$, and the tangent vector $x$ to the linear space $T_{q} \mathbb{E}=\mathbb{E}$ tangent at $q$ to $\mathbb{E}$. Later, with constraints determining a configuration space $Q$, the tangent bundle $T Q$ will be $Q \times \mathbb{E}$. See Abraham and Marsden (1978), Section 1.6.
} 


$$
\begin{aligned}
& m^{b}=m e^{b}: T_{q} \mathbb{E} \rightarrow T_{q}^{*} \mathbb{E} \\
& m^{\#}=\frac{1}{m} e^{\#}=\left(m^{b}\right)^{-1}: T_{q}^{*} \mathbb{E} \rightarrow T_{q} \mathbb{E} \\
& K=\frac{1}{2}\left\langle m^{b} \dot{q}, \dot{q}\right\rangle=\frac{1}{2} m\left\langle e^{b} \dot{q}, \dot{q}\right\rangle .
\end{aligned}
$$

Both momenta still coincide: $p=\dot{q}^{b}=d L_{q}(\dot{q})=m e^{b} \dot{q}$.

For $N$ particles with masses $m_{1}, \ldots, m_{N}$ moving freely in the Euclidean spaces $\mathbb{E}_{1}, \ldots, \mathbb{E}_{N}$ we have the kinetic energy

$$
K=\frac{1}{2} \sum_{n=1}^{N} m_{n}\left\langle e_{n}^{b} \dot{q}_{n}, \dot{q}_{n}\right\rangle
$$

where $e_{n}^{b}: T_{q} \mathbb{E}_{n} \rightarrow T_{q}^{*} \mathbb{E}_{n}$ and $\dot{q}_{n} \in T_{q} \mathbb{E}_{n} \quad(\forall n, q)$. We can also write $K=\left\langle m^{b} \dot{q}, \dot{q}\right\rangle / 2$, where the mass tensor

$$
\begin{aligned}
& m^{b}=\sum_{n=1}^{N} m_{n} e_{n}^{b}: T_{q} \mathbb{E} \rightarrow T_{q}^{*} \mathbb{E}, \\
& \mathbb{E}=\underset{n=1}{\times} \mathbb{E}_{n} \text { and } \dot{q}=\bigoplus_{n=1}^{N} \dot{q}_{n} .
\end{aligned}
$$

The inverse of $m^{b}$ is

$$
m^{\sharp}=\left(m^{b}\right)^{-1}=\sum_{n=1}^{N} \frac{1}{m_{n}} e_{n}^{\#}: T_{q}^{*} \mathbb{E} \rightarrow T_{q} \mathbb{E} .
$$

Here the level surfaces of $L_{q}$ are spherical with respect to $m^{b}$ but ellipsoidal when referred to

$$
e^{b}=\sum_{n=1}^{N} e_{n}^{b}: T_{q} \mathbb{E} \rightarrow T_{q}^{*} \mathbb{E}
$$

where the ratios of the masses are also those of the principal axes. The momentum $m^{b} \dot{q}$ remains a fairly direct descendant of what Descartes was groping for in Principia philosophice, and is still the same as the Lagrangian momentum $d L_{q}(\dot{q})=d K_{q}(\dot{q})$.

Now suppose holonomic, ${ }^{11}$ scleronomic ${ }^{12}$ constraints determine a Riemannian configuration space $Q \subset \mathbb{E}$. At each point $q \in Q$, the tangent space $T_{q} Q$ is contained in $T_{q} \mathbb{E}$. The mappings $\underline{e}^{b}: T_{q} Q \rightarrow T_{q}^{*} Q$ and $e^{b}: T_{q} \mathbb{E} \rightarrow T_{q}^{*} \mathbb{E}$ agree on all vectors in $T_{q} Q$ (but of course some velocities of $T_{q} \mathbb{E}$ are ruled out by the constraints), so $\underline{e}^{b}=\left.e^{b}\right|_{T_{q} Q}$. With masses we can write $\underline{m}^{b}=\left.m^{b}\right|_{T_{q} Q}$. Still no difference between the two momenta; we again have $p=\dot{q}^{b}=d L_{q}(\dot{q})=\underline{m}^{q} \dot{q}$.

So far there has been no potential, and $L=K$. A potential $U(q)$ depending on position alone will not contribute to the geometry, for with a Lagrangian $L(q, \dot{q})=K(q, \dot{q})-U(q)$ the differential $d L_{q}=d K_{q}-d U_{q}$ will just be $d K_{q}$. But if the

\footnotetext{
${ }^{11}$ See Lanczos (1970), pp.24-7.

${ }^{12}$ See Lanczos (1970), pp.31-4.
} 
potential depends on velocity as well, $d L_{q}$ will not be equal to $d K_{q}$, and there may be no tensor $\underline{m}^{b}: T_{q} Q \rightarrow T_{q}^{*} Q$ with the same effect as $d L_{q}$. In other words if the Lagrangian is the difference $K(q, \dot{q})-U(q, \dot{q})$, the level surfaces of $L_{q}$ may not even be ellipsoidal with respect to $\underline{e}^{b}$. So, for instance, the potential ${ }^{13} U=\phi+\left\langle A^{\sharp}, \dot{q}\right\rangle$ for a (unit) charge moving in an electromagnetic field characterized by the scalar potential $\phi$ and vector potential $A$ will make the Cartesian and Lagrangian momenta differ.

We now have three kinds of mechanical ingredients - constraints, masses, forces (potential)—and can introduce a corresponding nomenclature.

\begin{tabular}{|l|l|}
\hline GEOMETRY & TRANSFORMATION \\
\hline Empty & $e^{b}=d K_{q}=d L_{q}$ \\
\hline $\begin{array}{l}\text { Background } \\
\text { (constraints) }\end{array}$ & $\underline{e}^{b}=d K_{q}=d L_{q}$ \\
\hline $\begin{array}{l}\text { Kinematical } \\
\text { (constraints, masses) }\end{array}$ & $\underline{m}^{b}=d K_{q}=d L_{q}$ \\
\hline $\begin{array}{l}\text { Dynamical } \\
\text { (constraints, masses, forces) }\end{array}$ & $d L_{q}$ \\
\hline
\end{tabular}

The masses here are kinematical as they are seen as calibrating distance.

With a potential depending on position alone, $d K_{q}$ and $d L_{q}$ are the same, and hence the kinematical and dynamical geometries coincide. But a velocity-dependent potential can produce a dynamical geometry that differs from the kinematical.

Attitude to geometry can be conditioned by ontological prejudice: if constraints and masses are seen as mechanically primary, and forces as somewhat alien, it may be felt that the geometry of mechanics should be fundamentally kinematical. But then with velocity-dependent forces there will be two competing geometries, as can be illustrated with Hamilton-Jacobi theory.

\section{Perpendicularity in Hamilton-Jacobi theory}

A central feature of Hamilton-Jacobi theory ${ }^{14}$ is the interplay between a 'congruence' of (basically ${ }^{15}$ ) non-crisscrossing curves on the configuration space $Q$, and the level surfaces of the characteristic function $S: Q \rightarrow \mathbb{R}$. The relationship, determined by a transformation $T_{q}^{*} Q \rightarrow T_{q} Q \quad(\forall q \in Q)$ turning a covector field $d S=p$ into a vector field $\dot{q}$ tangent to a congruence on $Q$, can again be Cartesian and merely kinematical or Lagrangian and dynamical. Given a characteristic function $S$, a mass tensor $m^{\#}$ transforms the momentum $d S$ into the 'Cartesian' velocity $\underline{m}^{\sharp}(d S) \in T_{q} Q$, which will

\footnotetext{
${ }^{13}$ The velocity of light does not figure as it is set equal to one.

${ }^{14}$ For an account of the theory see Abraham and Marsden (1978) Section 5.2, Hermann (1968), or Lanczos (1970) pp.229-90.

${ }^{15}$ Focal or conjugate points are not the issue here.
} 
be the same as the 'Lagrangian' velocity $d H_{q}(d S)$ as long as the Hamiltonian is of the form $H=T+U(q)$, in which case we can write $\dot{q}=\underline{m}^{\sharp}(d S)=d H_{q}(d S)$.

Statements one comes across like "Nor is a mechanical path always perpendicular to the surfaces $S=$ const. An electron moving in a magnetic field does not cross the surfaces $S=$ const. perpendicularly"16 can be understood by mixing the Cartesian and Lagrangian pictures. The level surface $\bar{S}$ of $S$ at $q$ determines a ray $\bar{S}_{q} \subset T_{q}^{*} Q$, and any vector $p^{\#}$ dual to a covector $p \in \bar{S}_{q}$ will be 'perpendicular' to the level surface. But as this duality can be Cartesian or Lagrangian, the vector $d H_{q}(p)$ is necessarily perpendicular in the Cartesian sense neither to the ray $\bar{S}_{q}$ nor to the surface $\bar{S}$, for $\underline{m}^{\#}(p)$ is.

\section{Final remarks}

We have separated the geometrical contributions of the various mechanical ingredients: constraints, masses, forces. The "background" contribution made by constraints is not really the issue here, and was mentioned for completeness. Velocityindependent forces like gravity make no contribution at all. So we have been mainly interested in masses and velocity-dependent forces. The masses, or rather the mass tensor, gives rise to a kinematical geometry and to the momentum that came out of Descartes' 'theological' conservation principle. But the momentum associated here with Lagrange (and with a "dynamical" geometry) is more general, and cannot be based on a tensor in the presence of a velocity-dependent potential. Indeed the characteristic function in Hamilton-Jacobi theory can determine two congruences of trajectories, one Cartesian and kinematical, the other Lagrangian and dynamical.

I thank George Sparling and Hans Duistermaat for many fruitful discussions, and the Center for Philosophy of Science, University of Pittsburgh, for support of various kinds.

\section{References}

Abraham, R. and J. Marsden (1978) Foundations of mechanics, Cambridge, Perseus

Descartes, R. (1647) Principes de la philosophie, Paris, Henri le Gras

Hermann, R. (1968) Differential geometry and the calculus of variations, New York, Academic

Lanczos, C. (1970) The variational principles of mechanics, University of Toronto Press

\footnotetext{
${ }^{16}$ Lanczos (1970), p.267
} 